\title{
THE DERIVATIVE OF THE NEHARI FUNCTIONAL
}

\author{
Eric Schippers \\ University of Manitoba, Department of Mathematics \\ Winnipeg, Manitoba, R3T 2N2 Canada; eric_schippers@umanitoba.ca
}

\begin{abstract}
The Nehari functional assigns a number to subdomains of a fixed domain. For each choice of a certain singularity function, one obtains a different functional. In the case that the fixed outer domain is the unit disc, and the singularity is at the origin, we compute the derivative of Nehari's functional using a time-reversed Loewner equation and the power matrix. At an extremal, the derivative of Nehari's functional has a very simple form in terms of a quadratic differential associated with the singularity function.
\end{abstract}

\section{Introduction}

In this note it is shown that the derivative at an extremal of a functional of Nehari has a surprisingly simple expression in terms of a quadratic differential. The quadratic differential is naturally associated with the functional. The method of proof combines the Loewner method with Nehari's Dirichlet energy technique, with the help of the power matrix.

The Nehari functional, which assigns real numbers to subsets of the disc, is defined as follows in the case we consider here. Let

$$
x(z)=\sum_{k=-n}^{n} x_{k} z^{k}
$$

be such that $q=\operatorname{Re}(x)$ is zero on the boundary of the unit disc $\mathbf{D}$. For any simply connected domain $D_{1} \subset \mathbf{D}$ which is bounded by piecewise $C^{1}$ curves, let $q_{1}$ be the unique function on $D_{1}$ such that $q_{1}=0$ on the boundary $\partial D_{1}$ of $D_{1}$ and $q-q_{1}$ is harmonic on $D_{1}$. The Nehari functional is

$$
\operatorname{Neh}\left(D_{1}\right)=\int_{\partial D_{1}} q \frac{\partial q_{1}}{\partial n} d s
$$

In the case of equality, the domain $D_{1}$ is admissible for the quadratic differential $x^{\prime}(z)^{2} d z^{2}$ in the sense that the boundary is a trajectory.

Let $f: \mathbf{D} \rightarrow D_{1}$ be the bounded univalent function onto $\mathbf{D}$ satisfying $f(0)=0$ and $f^{\prime}(0)>0$ and let $y=x \circ f$. Let $p=1+p_{1} z+p_{2} z^{2}+\cdots \in \mathscr{P}$ where $\mathscr{P}$ is the set of holomorphic functions on $\mathbf{D}$ satisfying $\operatorname{Re}(p)>0$ and $p(0)=1$.

The results of this paper are the following.

(1) We derive a formula for the functional derivative of Neh at $D_{1}$ in the direction $p$ (Theorem 4.9).

doi:10.5186/aasfm. 2010.3518

2000 Mathematics Subject Classification: Primary 30C75, 30C55.

Key words: Dirichlet energy, Dirichlet space, bounded univalent functions, monotonicity, subordination, Loewner method, power matrix, extremal problems. 
(2) Let $f_{t}$ be a solution of $\dot{f}_{t}=-z p_{t} f_{t}$ with $p_{0}=p$ and $f_{0}=f$, where $\dot{f}_{t}$ denotes the derivative with respect to $t$. We show that the functional derivative of the Nehari functional at an extremal domain $f(\mathbf{D})$ in the direction specified by $p$ is

$$
\left.\frac{d}{d t}\right|_{t=0} \operatorname{Neh}\left(f_{t}(\mathbf{D})\right)=\lim _{r \rightarrow 1^{-}} \operatorname{Re} \frac{1}{\pi i} \int_{\gamma_{r}}\left(z y^{\prime}(z)\right)^{2} \cdot p(z) \cdot \frac{d z}{z},
$$

where $\gamma_{r}$ denotes the circle $|z|=r$ oriented positively (Corollary 4.11).

To differentiate the functional, we combine a time-reversed Loewner equation sometimes called the Friedland-Schiffer equation, with the Dirichlet energy method of Nehari. The computations are facilitated by the use of the power matrix, as in Schiffer and Tammi [6]. However, a simplification is obtained by our explicit use of the Lie algebra of the set of power matrices.

Unfortunately the use of the power matrix requires the introduction of some notation, and the reformulation of some standard results. However, this pays off as the use of the power matrix greatly simplifies the computation of the functional derivative. (The reader is invited to attempt to state and prove Theorem 4.9 without the power matrix). Furthermore, we can express Nehari's functional in an elegant form in terms of natural operators on extended Dirichlet space (Proposition 4.8).

Section 2 derives the necessary power matrix identities for one-parameter flows and the derivative of extended Dirichlet energy along a one-parameter flow. Section 3 proves a Green's identity for extended Dirichlet energy which is central to the proof of the main result. In Section 4.1, we state Nehari's theorem and show that without loss of generality the test function can be associated with a quadratic differential. The main results appear in Section 4, where we reformulate Nehari's functional in terms of the power matrix and differentiate the functional.

I am grateful to Oliver Roth for valuable discussions on this material. I am also grateful to the referee for suggestions which greatly improved the presentation of this paper.

\section{Preliminaries}

2.1. Vector and matrix notation for function spaces. Denote the Dirichlet energy of a holomorphic function $f$ on a open connected set $E \subset \mathbf{C}$ by

$$
\|f\|_{E}^{2}=\frac{1}{\pi} \iint_{E}\left|f^{\prime}\right|^{2}
$$

In particular, if $E=\mathbf{D}$ and $f(z)=\sum_{1}^{\infty} a_{k} z^{k}$, then

$$
\|f\|_{\mathbf{D}}^{2}=\sum_{k=1}^{\infty} k\left|a_{k}\right|^{2}
$$

Let $\mathscr{D}$ denote the Dirichlet space of holomorphic functions

$$
\mathscr{D}=\left\{f: \mathbf{D} \rightarrow \mathbf{C}:\|f\|_{\mathbf{D}}<\infty, f(0)=0\right\} .
$$

We will also consider an extension of the Dirichlet space

$$
\mathscr{D}_{-m}=\left\{f(z)=\sum_{k=-m}^{\infty} a_{k} z^{k} \mid \sum_{k=1}^{\infty} a_{k} z^{k} \in \mathscr{D}, f(0)=0\right\}
$$


and the truncated space

$$
\mathscr{D}_{-m}^{m}=\left\{f(z)=\sum_{k=-m}^{n} a_{k} z^{k}, f(0)=0\right\} .
$$

Define a degenerate inner product on $\mathscr{D}_{-m}^{m}$ by

$$
(f, g)=\sum_{k=-m}^{m} k \bar{a}_{k} b_{k}
$$

for $f(z)=\sum_{-m}^{m} a_{k} z^{k}$ and $g(z)=\sum_{-m}^{m} b_{k} z^{k}$. For $f, g \in \mathscr{D}_{-m}$, we will also write

$$
(f, g)=\sum_{k=-m}^{m} k \bar{a}_{k} b_{k}
$$

for the inner product of the truncations of $f$ and $g$ in $\mathscr{D}_{-m}^{m}$.

Denote the row vector consisting of the first coefficients of $f(z)=\sum_{k=-m}^{\infty} a_{k} z^{k} \in$ $\mathscr{D}_{-m}$ by

$$
\mathbf{f}=\left(a_{-n}, a_{-n+1}, \ldots, a_{n}\right)
$$

and write $(\mathbf{f}, \mathbf{g})=(f, g)$.

Remark 2.1. Although Nehari's functional is usually written in terms of Dirichlet energy, we found the most convenient expression for the present purposes to be in terms of extended Dirichlet energy (Proposition 4.8 ahead; see also [6]). In order to avoid confusion between the two, we will adopt the following convention: we will always use a norm $\|\cdot\|$ to refer to standard Dirichlet energy, and an inner product $(\cdot, \cdot)$ for extended Dirichlet energy.

Consider also the set of bounded univalent functions

$$
\mathscr{B}=\left\{f: \mathbf{D} \rightarrow \mathbf{D} \mid f \text { one-to-one, holomorphic, } f(0)=0, f^{\prime}(0)>0\right\} .
$$

$\mathscr{B}$ forms a group under composition. Given $f \in \mathscr{B}$ denote by $[f]$ the matrix whose entry in the $m$ th row and $n$th column is the $n$th coefficient of the power series of $f^{m}$ at 0 . We denote this coefficient by $[f]_{n}^{m}$. We adopt the convention that upper indices always denote row number and lower indices denote column number. Negative values of $m$ and $n$ are allowed, so the matrices are doubly infinite. It is easy to verify that composition of functions becomes matrix multiplication:

$$
[f \circ g]=[f][g] .
$$

Since the matrix is upper triangular for any $f$, multiplication involves only finite sums. Details on the power matrix can be found in $[2,6,7,8]$.

Let $f$ be a function holomorphic on $\mathbf{D}$ except for a pole of order $-m$ at 0 . $\mathscr{B}$ acts naturally on $f$ by composition on the right via $f \mapsto f \circ g$. If $f(z)=\sum_{-m}^{\infty} f_{k} z^{k}$, then the power series of $f \circ g$ is given by

$$
f \circ g(z)=\sum_{k=-m}^{\infty} f_{k} \sum_{l=k}^{\infty}[g]_{l}^{k} z^{l}=\sum_{l=-m}^{\infty} \sum_{k=-m}^{l} f_{k}[g]_{l}^{k} z^{l} .
$$

The infinitesimal generators of power matrices have a particularly simple representation. By differentiating a one-parameter family of matrices $\left[f_{t}\right]$, for which the 
corresponding family of functions satisfies $f_{0}(z)=z$ and $f_{t}=f_{0}+t h(z)+o(t)$ where $h(0)=0$, one can show that the derivative of $\left[f_{t}\right]$ at $t=0$ has the form

$$
\langle h\rangle_{n}^{m}=m h_{n-m+1} .
$$

Note that any holomorphic function $h(z)$ such that $h(0)=0$ is an infinitesimal generator; e.g. $f_{t}(z)=z+t h(z)$. We use the notation $\langle h\rangle$ to distinguish matrices of the form (2.3) from power matrices (note that $\langle h\rangle \neq[h]$ always).

We will need an identity for the coefficients of products and compositions [7]. It can be interpreted as representing the derivative of left multiplication.

Proposition 2.2. Let $h$ be a holomorphic function in a neighbourhood of 0 satisfying $h(0)=0$. Let $g$ be a holomorphic functions in a neighbourhood of 0 satisfying $g(0)=0$ and $g^{\prime}(0) \neq 0$. Then

$$
m\left[g^{m-1} g^{\prime} h\right]_{n}^{1}=\sum_{k}[g]_{k}^{m}\langle h\rangle_{n}^{k} .
$$

This identity was only stated in the case that $m \geq 0$ in [7]. The proofs given there are also valid for $m<0$ [8].

Remark 2.3. Since the power matrices are upper triangular, they behave nicely under truncation. That is, for any fixed $m$, if $-m \leq k \leq l \leq m$, then the $k, l$ th entry of $[f][g]$ is

$$
\sum_{j=l}^{k}[f]_{j}^{k}[g]_{l}^{j} .
$$

This sum depends only on the entries $[f]_{j}^{k}$ of $f$ and $[g]_{l}^{j}$ for which $n \leq j \leq m$. In other words, the multiplication formula $[f \circ g]=[f][g]$ continues to hold for the square blocks with rows and columns ranging between $-m$ and $m$. In the same way it can be shown that Proposition 2.2 continues to hold for such square blocks. We will not introduce new notation for these truncated power matrices. Thus by equation (2.2) we may for example write for $f \in \mathscr{D}_{-n}, g \in \mathscr{B}$,

$$
\mathbf{f} \circ \mathbf{g}=\mathbf{f}[g]
$$

where $\mathbf{f} \circ \mathbf{g}$ denotes the coefficient row vector of $f \circ g$ as above.

2.2. The Friedland-Schiffer equation and the power matrix. In this section, we derive formulas for the derivatives of power matrices of one-parameter flows of bounded univalent maps. These are used to derive simple expressions for the derivative of extended Dirichlet energy of one-parameter flows.

A time-reversed Loewner equation was introduced by Friedland and Schiffer:

$$
\dot{f}_{t}=-z p_{t}(z) f_{t}^{\prime}(z)
$$

where the dot denotes differentiation with respect to $t$, and $f_{t}$ is a one-parameter family of holomorphic functions. For $p_{t} \in \mathscr{P}$ measurable in $t, t \in[0, \infty)$, it can be shown that this differential equation with univalent initial condition $f_{0}: \mathbf{D} \rightarrow \mathbf{D}$ has a solution on $\mathbf{D}$ almost everywhere in $t[5]$. (Friedland and Schiffer prove this for $p_{t}$ which are extreme points of $\mathscr{P}$ [1].) The solution is an inward flow in the sense that $f_{t}(\mathbf{D}) \subset f_{s}(\mathbf{D})$ whenever $s \geq t$.

The power matrix of $f_{t}$ satisfying the Friedland-Schiffer equation satisfies the following simple differential equation. 
Proposition 2.4. Let $p_{t}(z) \in \mathscr{P}$ be measurable in $t$. Let $f_{t}$ be a solution to the Friedland-Schiffer equation $\dot{f}_{t}=-z p_{t}(z) f_{t}^{\prime}(z)$ on $[0, T]$ with infinitesimal generator $p_{t} \in \mathscr{P}$. Then for almost all $t \in \mathscr{P}$

$$
\frac{d}{d t}\left[f_{t}\right]=-\left[f_{t}\right]\left\langle z p_{t}\right\rangle
$$

Proof. This follows from Proposition 2.2 (see [7, 8]).

Remark 2.5. It follows from Remark 2.3 that Proposition 2.4 holds for truncated matrices.

Next we derive an expression for the derivative of extended Dirichlet energy $\left(y_{t}, y_{t}\right)$ for $y_{t}=x \circ f_{t}$, where $f_{t}$ is a one-parameter flow satisfying the FriedlandSchiffer equation. Some notation is necessary. Let $I^{\prime}$ be the diagonal matrix, all of whose diagonal entries are 1 with the exception of the 0,0 th entry which is zero. Note that

$$
\left(x, y I^{\prime}\right)=\left(x I^{\prime}, y\right)=(x, y)
$$

for any $x, y \in \mathscr{D}_{-n}^{n}$. Let $N$ be the $(2 n+1) \times(2 n+1)$ matrix with entries

$$
(N)_{j}^{i}= \begin{cases}0, & i \neq j \\ i, & i=j\end{cases}
$$

and let

$$
\left(N^{\prime}\right)_{j}^{i}= \begin{cases}0, & i \neq j \text { or } i=0 \\ 1 / i, & i=j .\end{cases}
$$

$N^{\prime}$ is the "inverse" of $N$ in the sense that $N^{\prime} N=N N^{\prime}=I^{\prime}$. Given an $(2 n+1) \times(2 n+1)$ matrix define

$$
A^{*}=N \overline{A^{T}} N^{\prime} .
$$

$A^{*}$ is the adjoint of $A$ in the following sense.

Proposition 2.6. For all $f, g \in \mathscr{D}_{-n}^{n}$

$$
\left(\mathbf{f} A^{*}, \mathbf{g}\right)=(\mathbf{f}, \mathbf{g} A) \text {. }
$$

Proof. It is clear that $(\mathbf{f}, \mathbf{g})=\mathbf{g} N \overline{\mathbf{f}}^{T}$, so

$$
(\mathbf{f}, \mathbf{g} A)=\mathbf{g} A N \overline{\mathbf{f}}^{T}=\mathbf{g} N N^{\prime} A N \overline{\mathbf{f}}^{T}=\mathbf{g} N{\overline{\mathbf{f} N \bar{A}^{T} N^{\prime}}}^{T}=\left(\mathbf{f} A^{*}, \mathbf{g}\right) .
$$

Proposition 2.7. Let $x \in \mathscr{D}_{-n}^{n}$ with $x(0)=0$. Let $f_{t}$ be a solution to the Friedland-Schiffer equation $\dot{f}_{t}=-z p_{t} f_{t}$, and $y_{t}=x \circ f_{t}$. Then

$$
\frac{d}{d t} \mathbf{y}_{t}=-\mathbf{y}_{t}\left\langle z p_{t}\right\rangle
$$

and

$$
\frac{d}{d t}\left(\mathbf{y}_{t}, \mathbf{y}_{t}\right)=-\left(\mathbf{y}_{t}, \mathbf{y}_{t}\left[\left\langle z p_{t}\right\rangle+\left\langle z p_{t}\right\rangle^{*}\right]\right)
$$

Proof. Since $\mathbf{x}$ and $\mathbf{y}$ are the first rows of a truncated power matrix, by Proposition 2.4 and the multiplicative property of power matrices under composition it follows that

$$
\frac{d}{d t} \mathbf{y}_{t}=\frac{d}{d t} \mathbf{x}\left[f_{t}\right]=\mathbf{x} \frac{d}{d t}\left[f_{t}\right]=-\mathbf{x}\left[f_{t}\right]\left\langle z p_{t}\right\rangle=-\mathbf{y}_{t}\left\langle z p_{t}\right\rangle
$$


It is then easy to compute that

$$
\frac{d}{d t}\left(\mathbf{y}_{t}, \mathbf{y}_{t}\right)=-\left(\mathbf{y}_{t}, \mathbf{y}_{t}\left\langle z p_{t}\right\rangle\right)-\left(\mathbf{y}_{t}\left\langle z p_{t}\right\rangle, \mathbf{y}_{t}\right)
$$

Remark 2.8. The right hand side is $-2 \operatorname{Re}\left(\mathbf{y}_{t}, \mathbf{y}_{t}\langle z p\rangle\right)$, and in particular is real (as it must be).

\section{A Green's identity for extended Dirichlet energy}

Because elements of $\mathscr{D}_{-n}^{n}$ can have a singularity at 0 , one cannot apply the complex form of Green's theorem directly to convert extended Dirichlet energy to a contour integral. In this section we derive an identity which replaces Green's theorem in this general setting.

For $w \in \mathscr{D}_{-n}^{n}$ let

$$
w_{+}=\sum_{k=1}^{n} w_{k} z^{k}
$$

and

$$
w_{-}=\sum_{k=-n}^{-1} w_{k} z^{k}
$$

Denote the reflection of a function in the circle by

$$
w^{*}(z)=\overline{w(1 / \bar{z})} \text {. }
$$

It is easy to verify that for $w, v \in \mathscr{D}_{-n}^{n}$

$$
(w, v)=\sum_{k=1}^{n} k \overline{w_{k}} v_{k}-\sum_{k=-n}^{-1}|k| \overline{w_{k}} v_{k}=\left(w_{+}, v_{+}\right)-\overline{\left(w_{-}^{*}, v_{-}^{*}\right)}
$$

Note that both terms on the far right hand side of equation (3.2) are Dirichlet integrals of analytic functions without singularities.

Below, for $F \in \mathscr{D}_{-n}$ let $\{F\}_{n}$ denote the truncation of $F$ in $\mathscr{D}_{-n}^{n}$.

Proposition 3.1. For $w, v \in \mathscr{D}_{-n}^{n}$ and $p$ analytic in $\mathbf{D}$,

$$
\left(w, z p v^{\prime}\right)=\frac{1}{2 \pi i} \int_{\partial \mathbf{D}}\left(z w^{\prime}\right)^{*}(z)\left\{z v^{\prime}(z) p(z)\right\}_{n} \frac{d z}{z} .
$$

Furthermore, the right hand side can be written

$$
\left(w, z p v^{\prime}\right)=\frac{1}{2 \pi i} \int_{\partial \mathbf{D}}\left(z w^{\prime}\right)^{*}(z) \cdot z v^{\prime}(z)\{p(z)\}_{2 n} \frac{d z}{z} .
$$

Proof. Below we denote the positive part of the truncated function $\left\{z p v^{\prime}\right\}_{n}$ by $\left(z p v^{\prime}\right)_{+}$. By the complex form of Green's identity

$$
\left(w_{+},\left(z p v^{\prime}\right)_{+}\right)=\frac{1}{\pi} \int_{\mathbf{D}} \overline{w_{+}^{\prime}(z)}\left(z p v^{\prime}\right)_{+}^{\prime}(z) \frac{d \bar{z} \wedge d z}{2 i}=-\frac{1}{2 \pi i} \int_{\partial \mathbf{D}} \overline{w_{+}^{\prime}(z)}\left(z p v^{\prime}\right)_{+}(z) d \bar{z} .
$$

Since $d \bar{z}=-\bar{z} d z / z$ and $z=1 / \bar{z}$ on the region of integration,

$$
\begin{aligned}
\left(w_{+},\left(z p v^{\prime}\right)_{+}\right) & =\frac{1}{2 \pi i} \int_{\partial \mathbf{D}} \overline{\left(z w^{\prime}\right)_{+}(z)}\left(z p v^{\prime}\right)_{+}(z) \frac{d z}{z} \\
& =\frac{1}{2 \pi i} \int_{\partial \mathbf{D}} \overline{\left(z w^{\prime}\right)_{+}(1 / \bar{z})}\left(z p v^{\prime}\right)_{+}(z) \frac{d z}{z}
\end{aligned}
$$


Applying the complex form of Green's identity to the second term in equation $(3.2)$

$$
\begin{aligned}
\overline{\left(\overline{w_{-}(1 / \bar{z})}, \overline{\left(z p v^{\prime}\right)_{-}(1 / \bar{z})}\right)} & =\frac{1}{\pi} \overline{\iint_{\mathbf{D}} \overline{\frac{\partial}{\partial z} \overline{w_{-}(1 / \bar{z})}} \frac{\partial}{\partial z} \overline{\left(z p v^{\prime}\right)_{-}(1 / \bar{z})} \frac{d \bar{z} \wedge d z}{2 i}} \\
& =\frac{1}{2 \pi i} \iint_{\mathbf{D}} \frac{\partial}{\partial z} \overline{w_{-}(1 / \bar{z})} \frac{\partial}{\partial \bar{z}}\left[\left(z p v^{\prime}\right)_{-}(1 / \bar{z})\right] d \bar{z} \wedge d z \\
& =\frac{1}{2 \pi i} \int_{\mathbf{D}} \frac{\partial}{\partial z} \overline{w_{-}(1 / \bar{z})}\left(z p v^{\prime}\right)_{-}(1 / \bar{z}) d z
\end{aligned}
$$

Again using the fact that $|z|^{2}=1$ on $\partial \mathbf{D}$,

$$
\begin{aligned}
\overline{\left(\overline{w_{-}(1 / \bar{z})}, \overline{\left(z p v^{\prime}\right)_{-}(1 / \bar{z})}\right)} & =-\frac{1}{2 \pi i} \int_{\partial \mathbf{D}} \overline{\overline{\bar{z}}} \overline{\bar{z}} w_{-}^{\prime}(1 / \bar{z})\left(z p v^{\prime}\right)_{-}(1 / \bar{z}) \frac{d z}{z} \\
& =-\frac{1}{2 \pi i} \int_{\partial \mathbf{D}} \overline{\left(z w^{\prime}\right)_{-}(1 / \bar{z})}\left(z p v^{\prime}\right)_{-}(z) \frac{d z}{z}
\end{aligned}
$$

The first claim now follows from the observation that

$$
\int_{\partial \mathbf{D}} \overline{\left(z w^{\prime}\right)_{+}(1 / \bar{z})}\left(z p v^{\prime}\right)_{-}(z) \frac{d z}{z}=\int_{\partial \mathbf{D}} \overline{\left(z w^{\prime}\right)_{-}(1 / \bar{z})}\left(z p v^{\prime}\right)_{+}(z) \frac{d z}{z}=0
$$

by the residue theorem.

To prove the second claim, observe that if

$$
g=\sum_{k=-n}^{n} \in \mathscr{D}_{-n}^{n}
$$

then (adopting the convention that $g_{k}=0$ for $k>n$ )

$$
\begin{aligned}
\{g p\}_{n} & =\left\{\sum_{k=-n}^{\infty} \sum_{j=-n}^{k} g_{j} p_{n+j} z^{k}\right\}_{n}=\sum_{k=-n}^{n} \sum_{j=-n}^{k} g_{j} p_{n+j} z^{k} \\
& =\left\{\left(\sum_{k=-n}^{n} g_{k} z^{k}\right)\left(\sum_{j=0}^{2 n} p_{j} z^{j}\right)\right\}_{n}=g\{p\}_{2 n} .
\end{aligned}
$$

Thus, if $f, g \in \mathscr{D}_{-n}^{n}$, then

$$
\int_{\partial \mathbf{D}} f\{g p\}_{n} \frac{d z}{z}=\int_{\partial \mathbf{D}} f g\{p\}_{2 n} \frac{d z}{z},
$$

from which the second claim follows.

This has a expression in terms of the Hardy space inner product, which does not explicitly involve truncation.

Corollary 3.2. Let $\gamma_{r}$ denote the curve $|z|=r$ with positive orientation. For $w, v \in \mathscr{D}_{-n}^{n}$ and $p$ analytic in $\mathbf{D}$,

$$
\left(w, z p v^{\prime}\right)=\lim _{r \rightarrow 1^{-}} \frac{1}{2 \pi i} \int_{\gamma_{r}}\left(z w^{\prime}\right)^{*}(z) \cdot z v^{\prime}(z) \cdot p(z) \frac{d z}{z} .
$$

Proof. This follows from the second part of Proposition 3.1 and the fact that $\left(z w^{\prime}\right)^{*}(z) \cdot z v^{\prime}(z)$ has only finitely many negative terms. 


\section{Nehari's functional and its derivative}

Nehari's monotonicity theorem associates a functional of pairs of nested domains to a test function $x$. We are concerned with the case that both domains are simply connected, the outer domain is $\mathbf{D}$, and $x$ is a holomorphic function on $\mathbf{D}$ except for a pole at the origin. In this section, we state Nehari's theorem, and rewrite it in terms of the power matrix. We then prove the main results on the derivative of Nehari's functional.

4.1. Nehari's monotonicity theorem. In this section, we rewrite Nehari's functional in terms of the coefficients of $x$, and show that without loss of generality the test function $x$ can be chosen in such a way that $x^{\prime 2} d z^{2}$ is a quadratic differential on the disc.

Let $D_{1} \subset \mathbf{D}$ be a simply connected domain. We will assume that $D_{1}$ is bounded by a piecewise $C^{1}$ curve. Let $q$ be a real-valued function on $\mathbf{D}$ which is harmonic except with specified singularities at a finite set of points $z_{k}$. That is, for some singularity function

$$
S(z)=\operatorname{Re} \sum_{k=1}^{n} \frac{\alpha_{k}}{\left(z-z_{k}\right)^{m_{k}}},
$$

let $q$ be the unique function such that $q=0$ on $\partial \mathbf{D}$ and $q+S$ is harmonic on $\mathbf{D}$. Similarly, let $q_{1}$ be the unique real-valued function such that $q_{1}=0$ on $\partial D_{1}$ and $q_{1}+S$ is harmonic on $D_{1}$.

Remark 4.1. Nehari allows $S$, and hence $q$, to have a logarithmic singularity. In this case $x$ will be multi-valued. We do not consider this case here.

We then have the following. Denote the Dirichlet energy of a real function over a domain $E$ by $\|\cdot\|_{E}$, i.e.

$$
\|q\|_{E}^{2}=\iint_{E}|\nabla q|^{2}
$$

Theorem 4.2. (Nehari monotonicity theorem) Let $S$ be a singularity function (4.1). Let $D_{1} \subset D_{2} \subset \mathbf{D}$, with $D_{1}$ and $D_{2}$ simply connected domains bounded by piecewise $C^{1}$ curves. Let $q_{1}, q_{2}$ and $q$ be the harmonic functions corresponding to $D_{1}, D_{2}$ and $\mathbf{D}$ respectively, as defined above. The inequality

$$
\int_{\partial D_{1}} S \frac{\partial q_{1}}{\partial n} d s \geq \int_{\partial D_{2}} S \frac{\partial q_{2}}{\partial n} d s
$$

holds, where $n$ denotes the outward unit normal and ds denotes infinitesimal arc length.

It follows from Theorem 4.2 that the lower bound of the functional

$$
\int_{\partial D_{1}} S \frac{\partial q_{1}}{\partial n} d s
$$

is attained by $D_{1}=\mathbf{D}$, that is

$$
\int_{\partial D_{1}} S \frac{\partial q_{1}}{\partial n} d s-\int_{\partial \mathbf{D}} S \frac{\partial q}{\partial n} d s \geq 0
$$

Clearly the left hand side of the above inequality is still monotonic. 
We will now give this functional a more convenient form. For $D_{1}, S, q$ and $q_{1}$ as above,

$$
\int_{\partial D_{1}} S \frac{\partial q_{1}}{\partial n} d s-\int_{\partial \mathbf{D}} S \frac{\partial q}{\partial n} d s=\int_{\partial D_{1}}-q \frac{\partial q_{1}}{\partial n} d s
$$

To see this, let

$$
u(z)= \begin{cases}S(z), & z \in \mathbf{D} \backslash D_{1}, \\ q_{1}(z)+S(z), & z \in D_{1},\end{cases}
$$

and

$$
v(z)=q(z)+S(z)-u(z)= \begin{cases}q(z), & z \in \mathbf{D} \backslash D_{1}, \\ q(z)-q_{1}(z), & z \in D_{1} .\end{cases}
$$

Since $v=0$ on $\partial \mathbf{D}$ and $q_{1}=0$ on $\partial D_{1}$, by Green's identity

$$
\iint_{\mathbf{D}} \nabla v \cdot \nabla(q+S) d A=0
$$

It follows that $\|u\|_{\mathbf{D}}^{2}-\|q+S\|_{\mathbf{D}}^{2}=\|v\|_{\mathbf{D}}^{2}$. Using Green's identity, it can be shown that

$$
\|u\|_{\mathbf{D}}^{2}-\|q+S\|_{\mathbf{D}}^{2}=\int_{\partial D_{1}} S \frac{\partial q_{1}}{\partial n} d s-\int_{\partial \mathbf{D}} S \frac{\partial q}{\partial n} d s .
$$

Again using Green's identity it is easily shown that

$$
\|v\|_{\mathbf{D}}^{2}=\int_{\partial D_{1}}-q \frac{\partial q_{1}}{\partial n} d s
$$

which completes the proof of equation (4.3).

Thus, by subtracting the lower bound

$$
\int_{\partial \mathbf{D}} S \frac{\partial q}{\partial n} d s
$$

and changing the sign, we may define Nehari's functional to be

$$
\operatorname{Neh}\left(D_{1}\right)=\int_{\partial D_{1}} q \frac{\partial q_{1}}{\partial n} d s
$$

and it holds that $D_{1} \subset D_{2} \Rightarrow \operatorname{Neh}\left(D_{1}\right) \leq \operatorname{Neh}\left(D_{2}\right)$. The upper bound of the functional is zero.

The function $q$ is associated with a quadratic differential on $\mathbf{D}$. Let $x$ be an analytic function such that $q=\operatorname{Re}(x)$. The condition that $q$ is constant is equivalent to the condition that $x^{\prime 2} d z^{2}$ is a quadratic differential admissible for the disc. To see this, note that $q$ is constant if and only if

$$
0=q_{x} d x+q_{y} d y=2 \operatorname{Re}\left(x^{\prime}(z) d z\right)=2 \operatorname{Re}\left(i z x^{\prime}(z) d \theta\right)
$$

(where $z=e^{i \theta}$ along the boundary of the disc) which holds if and only if $\operatorname{Im} z x^{\prime}(z)=$ 0 . Thus $\partial \mathbf{D}$ is a negative trajectory of $x^{\prime}(z)^{2} d z^{2}$. Assuming that $q$ is associated to a quadratic differential this way amounts to subtracting a constant and thus there is no loss of generality.

Remark 4.3. Setting $q=0$ and $q_{1}=0$ on the boundaries of $\mathbf{D}$ and $D_{1}$ is only a convention. The method only requires that they be constant on the boundary. Adding a constant to $q$ or $q_{1}$ doesn't change the inequality. 
Next we derive an expression for the Nehari functional in terms of the coefficients of $x$. We will henceforth assume that $x \in \mathscr{D}_{-n}^{n}$. Let $f: \mathbf{D} \rightarrow D_{1}$ be a one-to-one onto map satisfying $f(0)=0$. It is necessary to find the relation between the functions $y=x \circ f$ and $q_{1}$. We claim that if $q=\operatorname{Re} x$ and $D_{1}=f(\mathbf{D})$, then

$$
q_{1} \circ f=\operatorname{Re}\left(\sum_{k=-n}^{-1}\left(y_{k} z^{k}-\bar{y}_{k} z^{-k}\right)\right) \text {. }
$$

Let $h(z)$ denote the right hand side of the above equation. First, note that $h(z)$ is zero on $\partial \mathbf{D}$ since $\bar{z}^{k}=z^{-k}$. Also $h(z)-\operatorname{Re} x \circ f$ is non-singular on $\mathbf{D}$. Thus $h \circ f^{-1}=q_{1}$ which proves the claim. Furthermore, we have that

$$
q \circ f-q_{1} \circ f=\operatorname{Re}\left(\sum_{k=n+1}^{\infty} y_{k} z^{k}+\sum_{k=1}^{n}\left(y_{k}+\bar{y}_{k}\right) z^{k}\right) .
$$

Thus we have

$$
\begin{aligned}
\|q\|_{\mathbf{D} \backslash D_{1}}^{2}+\left\|q-q_{1}\right\|_{D_{1}}^{2} & =\|q\|_{\mathbf{D} \backslash D_{1}}^{2}+\left\|q \circ f-q_{1} \circ f\right\|_{\mathbf{D}}^{2} \\
& =\|x\|_{\mathbf{D} \backslash D_{1}}^{2}+\left\|\sum_{k=n+1}^{\infty} y_{k} z^{k}\right\|_{\mathbf{D}}^{2}+\left\|\sum_{k=1}^{n}\left(y_{k}+\bar{y}_{-k}\right) z^{k}\right\|_{\mathbf{D}}^{2} \\
& =(x, x)-(y, y)+\left\|\sum_{k=1}^{n}\left(y_{k}+\bar{y}_{-k}\right) z^{k}\right\|_{\mathbf{D}}^{2} .
\end{aligned}
$$

The last step follows from a simple computation using Green's identity [6, pp. 4-5]:

$$
\|x\|_{\mathbf{D} \backslash D}^{2}=\sum_{k=-n}^{n} k\left|x_{k}\right|^{2}-\sum_{k=-n}^{\infty} k\left|y_{k}\right|^{2} .
$$

In summary, in the case that the outer domain is $\mathbf{D}$, the inner domain is simply connected, and the test function has a pole at 0 , we may formulate Nehari's theorem as follows.

Theorem 4.4. (Reformulation of Nehari's inequalities) Let $x \in \mathscr{D}_{-n}^{n}$ be such that $x^{\prime}(z)^{2} d z^{2}$ is a quadratic differential on the disc (that is, $x^{\prime}(z)^{2} d z^{2} \leq 0$ on $\partial \mathbf{D}$ ). Let $D_{1} \subset \mathbf{D}$ be a simply connected domain bounded by a piecewise $C^{1}$ curve. Let $f: \mathbf{D} \rightarrow D_{1}$ be a univalent function such that $f(0)=0$ and $f^{\prime}(0)>0$, and let $y=x \circ f$. The functional

$$
\operatorname{Neh}\left(D_{1}\right)=(x, x)-(y, y)+\left\|\sum_{k=1}^{n}\left(y_{k}+\bar{y}_{-k}\right) z^{k}\right\|_{\mathbf{D}}^{2}
$$

is such that $D_{1} \subset D_{2} \Rightarrow \operatorname{Neh}\left(D_{1}\right) \leq \operatorname{Neh}\left(D_{2}\right)$. The upper bound of the functional is zero.

The preceding paragraphs show that there is no loss of generality in assuming that $x^{\prime 2} d z^{2}$ is a quadratic differential on $\mathbf{D}$.

Of course we may view Neh as a function of $D_{1}, f$ or $y$. Note that since the expression for Neh given in equation (4.4) only depends on the domain $D_{1}$ and not on $f$, expression (4.7) must be invariant under rotations $f \mapsto e^{i \theta} f$. Thus the normalization $f^{\prime}(0)>0$ is inconsequential, and only serves to uniquely determine $f$. 
4.2. The case of equality. In this section we show that equality is attained in Neh if and only if $y=x \circ f$ is such that $y^{\prime}(z)^{2} d z^{2}$ is a quadratic differential which is admissible for the disc in the sense that $\partial \mathbf{D}$ is a negative trajectory. Equivalently, $f$ maps $\mathbf{D}$ onto the disc minus trajectories of $x^{\prime}(z)^{2} d z^{2}$.

Let $y \in \mathscr{D}_{-n}$. The boundary of $\mathbf{D}$ is a negative trajectory of $y^{\prime}(z)^{2} d z^{2}$ if and only if $z^{2} y^{\prime}(z)^{2} \geq 0$ on $\partial \mathbf{D}$, which holds if and only if $\operatorname{Im}\left(z y^{\prime}(z)\right)=0$ for all $z \in \mathbf{D}$. Thus

$$
y^{\prime}(z)^{2} d z^{2} \leq 0 \text { on } \partial \mathbf{D} \Leftrightarrow y_{k}=-\bar{y}_{-k}, k=1, \ldots, n \text { and } y_{k}=0, k \geq n+1 .
$$

The upper bound of the Nehari functional is zero, which is attained by $\mathbf{D}$ for every choice of function $x$. It is also attained by any domain $D_{1}$ which is admissible for the quadratic differential $x^{\prime 2} d z^{2}$, and these are the only domains for which this is true. This can be read easily from the second equality of equation (4.6). If the Nehari functional is zero, we must have that

(1) $\|x\|_{\mathbf{D} \backslash D_{1}}^{2}=0$, so $\mathbf{D} \backslash D_{1}$ has measure zero,

(2) $\left\|\sum_{k=n+1}^{\infty} y_{k} z^{k}\right\|_{\mathrm{D}}^{2}=0$, so $y_{k}=0$ for $k \geq n+1$,

(3) $\left\|\sum_{k=1}^{n}\left(y_{k}+\bar{y}_{-k}\right) z^{k}\right\|_{\mathbf{D}}^{2}=0$ so $y_{k}=-\bar{y}_{-k}$ for $k=1, \ldots, n$.

So by (4.8) this occurs if and only if $\partial \mathbf{D}$ is a negative trajectory of $y^{\prime}(z)^{2} d z^{2}$. This implies that $D_{1}=f(\mathbf{D})$ is admissible for $x^{\prime}(z)^{2} d z^{2}$. By admissible, we mean that $D_{1}$ is $\mathbf{D}$ minus arcs of negative trajectories of $x^{\prime}(z)^{2} d z^{2}$.

Conversely, if $D_{1}$ is admissible for $x^{\prime}(z)^{2} d z^{2}, f: \mathbf{D} \rightarrow D_{1}$ is the normalized mapping function, and $y=x \circ f$, then condition (1) above is satified. Furthermore, $\mathbf{D}$ is admissible for $y^{\prime 2}(z) d z^{2}$. This implies that $y_{k}=-\bar{y}_{-k}$ and, in particular, $y_{k}=0$ for $k>n$. Thus conditions (2) and (3) are satisfied, and so $D_{1}$ is extremal for the Nehari functional.

Remark 4.5. Although here Nehari's functional has only been defined for domains bounded by piecewise $C^{1}$ curves, it can be extended to all simply connected subdomains of $\mathbf{D}$ for example by expressing it in terms of the coefficients of $x$ and $f$. The inequality can likewise be extended. In this case one can apply Schiffer variation to show that the extremals domains must map onto $\mathbf{D}$ minus curves which are sufficiently regular to apply the above argument. So there are no extremals for the extended functional other than those found above.

4.3. Derivative of the Nehari functional. In order to compute the derivative of the Nehari functional, we introduce two convenient operations on vectors: a reflection and the Hilbert transform. We will adopt the convention that operators act on vectors on the right.

Remark 4.6. This convention is necessary, if we use row vectors to denote elements of $\mathscr{D}_{-n}^{n}$. Using row vectors is in turn forced on us by the power matrix multiplication formula, and the fact that composition acts on the right.

Define the reflection operator $R$ on vectors by

$$
\left(y_{-n}, y_{-n+1}, \ldots, y_{n-1}, y_{n}\right) R=\left(\bar{y}_{n}, \bar{y}_{n-1}, \ldots, \bar{y}_{-n}\right) .
$$

This corresponds to reflecting the function $y(z)$ in the unit circle. That is, if

$$
g(z)=y^{*}(z)=\overline{y(1 / \bar{z})},
$$

then

$$
\mathrm{g}=\mathrm{y} R
$$


It has the following easily verified properties:

$$
(\mathbf{v}, \mathbf{w} R)=-\overline{(\mathbf{v} R, \mathbf{w})}
$$

since

$$
(\mathbf{v}, \mathbf{w} R)=\sum_{k=-n}^{n} k \bar{v}_{k} \bar{w}_{-k}=\sum_{k=-n}^{n}-k \bar{v}_{-k} \bar{w}_{k}=-\overline{(\mathbf{v} R, \mathbf{w})} .
$$

Clearly $R^{2}$ is the identity. It follows immediately from equation (4.10) that

$$
(\mathbf{v} R, \mathbf{w} R)=-\overline{(\mathbf{v}, \mathbf{w})} \text { and }(\mathbf{w} R, \mathbf{w} R)=-(\mathbf{w}, \mathbf{w}),
$$

so furthermore

$$
(\mathbf{w}, \mathbf{w} R)=0 .
$$

We thus also have that

$$
(\mathbf{w}+\mathbf{w} R, \mathbf{w}+\mathbf{w} R)=0 .
$$

We need the action of $R$ on matrices $\langle z p\rangle$ of infinitesimal generators.

Proposition 4.7. For matrices of the form $\langle z p\rangle$,

$$
\langle z p\rangle R I^{\prime}=-\langle z p\rangle^{*} I^{\prime}
$$

Proof. Define the flip operator on square matrices whose entries range from $-n$ to $n$ :

$$
A^{f}{ }_{i j}=\overline{A_{-i-j}} \text {. }
$$

With this definition

$$
(\mathbf{v} A) R=\mathbf{v} R A^{f} .
$$

To see this compute

$$
\begin{aligned}
(\mathbf{v} A) R & =\left(\sum_{k=-n}^{n} v_{k} A_{k-n}, \ldots, \sum_{k=-n}^{n} v_{k} A_{k n}\right) R=\left(\sum_{k=-n}^{n} \overline{v_{k}} \overline{A_{k n}}, \ldots, \sum_{k=-n}^{n} \overline{v_{k}} \overline{A_{k-n}}\right) \\
& =\left(\sum_{k=-n}^{n} \overline{v_{-k}} \overline{A_{-k n}}, \ldots, \sum_{k=-n}^{n} \overline{v_{-k}} \overline{A_{-k-n}}\right)=\mathbf{v} R A^{f} .
\end{aligned}
$$

By equations (2.6) and (2.3) it can be computed that

$$
\langle z p\rangle_{k j}^{*}= \begin{cases}k \overline{p_{k-j}}, & j \leq k, j \neq 0 \\ 0, & \text { otherwise. }\end{cases}
$$

On the other hand

$$
\langle z p\rangle_{k j}^{f}= \begin{cases}-k \overline{p_{k-j}}, & j \leq k \\ 0, & \text { otherwise }\end{cases}
$$

which proves the claim.

Next we define the operator

$$
\left(y_{-n}, \ldots, y_{n}\right) J=\left(-y_{n},-y_{-n+1}, \ldots,-y_{-1}, 0, y_{1}, \ldots, y_{n}\right) .
$$

The operator $i J$ is the Hilbert transform. It is clear that

$$
(\mathbf{w} J) R=-\mathbf{w} R J,
$$




$$
J^{2}=I^{\prime}
$$

and

$$
(\mathbf{v} J, \mathbf{w})=(\mathbf{v}, \mathbf{w} J) .
$$

We are now prepared to differentiate the Nehari functional. Let $f_{t}$ satisfy the Friedland-Schiffer equation for some $p_{t}$ and initial condition $f_{0}(z)=z$, and let $y_{t}=$ $x \circ f_{t}$. Recall that the Nehari functional is

$$
\operatorname{Neh}\left(f_{t}(\mathbf{D})\right)=(\mathbf{x}, \mathbf{x})-\left(\mathbf{y}_{t}, \mathbf{y}_{t}\right)+\left\|\sum_{k=1}^{n}\left(y_{k}+y_{-k}\right) z^{k}\right\|_{\mathbf{D}}^{2}
$$

We now write the Nehari functional in a more convenient form.

Proposition 4.8. The Nehari functional can be written in the following form,

$$
\operatorname{Neh}\left(f_{t}(\mathbf{D})\right)=(\mathbf{x}, \mathbf{x})-\operatorname{Re}\left(\mathbf{y}_{t}(I+R), \mathbf{y}_{t}(I-J)\right) \text {. }
$$

Proof. We will temporarily suppress the subscript $t$ to reduce clutter. It is clear that

$$
\sum_{k=1}^{n}\left(y_{k}+\overline{y_{-k}}\right) z^{k}=\frac{1}{2}[(\mathbf{y}+\mathbf{y} R)+(\mathbf{y}+\mathbf{y} R) J] I^{\prime} .
$$

From equations (4.13) and (4.18) it follows that

$$
((\mathbf{y}+\mathbf{y} R) J,(\mathbf{y}+\mathbf{y} R) J)=(\mathbf{y}+\mathbf{y} R, \mathbf{y}+\mathbf{y} R)=0
$$

so

$$
\left\|\sum_{k=1}^{n}\left(y_{k}+y_{-k}\right) z^{k}\right\|^{2}=\frac{1}{2} \operatorname{Re}(\mathbf{y}+\mathbf{y} R,(\mathbf{y}+\mathbf{y} R) J) .
$$

By equations (4.16) and (4.11) it follows that

$$
(\mathbf{y}, \mathbf{y} J)=-\overline{(\mathbf{y} R, \mathbf{y} J R)}=\overline{(\mathbf{y} R, \mathbf{y} R J)} \text {. }
$$

By equations (4.16) and (4.10),

$$
(\mathbf{y}, \mathbf{y} R J)=\overline{(\mathbf{y} R, \mathbf{y} J)}
$$

Applying these last two equations to (4.19) proves the claim.

We are now ready to differentiate the Nehari functional. Let $[A, B]=A B-B A$ denote the matrix commutator.

\section{Theorem 4.9.}

$\frac{d}{d t} \operatorname{Neh}\left(f_{t}(\mathbf{D})\right)=-\operatorname{Re}\left(\mathbf{y}_{t}+\mathbf{y}_{t} R, \mathbf{y}_{t}[(I-J),\langle z p\rangle]\right)+\operatorname{Re}\left(\mathbf{y}_{t}, \mathbf{y}_{t}\left(\langle z p\rangle+\langle z p\rangle^{*}\right)(I-J)\right)$.

Proof. By Propositions 2.7 and 4.7 and equation (4.15),

$$
\frac{d}{d t} \mathbf{y} R=-\mathbf{y}\langle z p\rangle R=\mathbf{y} R\langle z p\rangle^{*}
$$

(again suppressing $t$ ) and clearly

$$
\frac{d}{d t} \mathbf{y} J=-\mathbf{y}\langle z p\rangle J
$$


So using the second expression of Proposition 4.8

$$
\begin{aligned}
\frac{d}{d t} \operatorname{Neh}\left(f_{t}(\mathbf{D})\right)= & -\operatorname{Re}\left(-\mathbf{y}\langle z p\rangle+\mathbf{y} R\langle z p\rangle^{*}, \mathbf{y}(I-J)\right)+\operatorname{Re}(\mathbf{y}+\mathbf{y} R, \mathbf{y}\langle z p\rangle(I-J)) \\
= & \operatorname{Re}(\mathbf{y}\langle z p\rangle, \mathbf{y}(I-J))-\operatorname{Re}(\mathbf{y} R, \mathbf{y}(I-J)\langle z p\rangle) \\
& -\operatorname{Re}(\mathbf{y}, \mathbf{y}(I-J)\langle z p\rangle)+\operatorname{Re}\left(\mathbf{y}\langle z p\rangle^{*}, \mathbf{y}(I-J)\right) \\
& +\operatorname{Re}(\mathbf{y}+\mathbf{y} R, \mathbf{y}\langle z p\rangle(I-J)),
\end{aligned}
$$

where we have inserted the two terms

$$
0=-\operatorname{Re}(\mathbf{y}, \mathbf{y}(I-J)\langle z p\rangle)+\operatorname{Re}\left(\mathbf{y}\langle z p\rangle^{*}, \mathbf{y}(I-J)\right)
$$

Collecting terms in equation (4.20) and using the fact that $I-J$ is self-adjoint, the claim follows.

In the special case that the domain $D_{1}$ is extremal and hence admissible for the fixed quadratic differential $x^{\prime 2} d z^{2}$, the derivative of the Nehari functional has a particularly nice form.

Corollary 4.10. Let $x \in \mathscr{D}_{-n}^{n}$ be such that $x^{\prime}(z) d z^{2}$ is a quadratic differential on the disc (i.e. such that $x_{k}=-\overline{x_{-k}}$ ). Let $f_{t}: \mathbf{D} \rightarrow \mathbf{D}$ satisfy the Friedland-Schiffer equation for some $p_{t}$ and let $y_{t}=x \circ f_{t}$. If $f_{t_{0}}$ is admissible for this quadratic differential for some fixed $t_{0}$ then

$$
\left.\frac{d}{d t}\right|_{t=t_{0}} \operatorname{Neh}\left(f_{t}(\mathbf{D})\right)=2 \operatorname{Re}\left(\mathbf{y}_{t_{0}}, \mathbf{y}_{t_{0}}\left\langle z p_{t_{0}}\right\rangle\right)
$$

Proof. We suppress $t$ subscripts as usual. The condition that $f_{t_{0}}$ be admissible implies that $y_{k}=0$ for $k>n$ and $y_{k}=-\overline{y_{-k}}$, that is $\mathbf{y} R=-\mathbf{y}$. Thus the first term of Theorem 4.9 vanishes. Furthermore, by Proposition 4.7 and equations (4.10) and (4.16),

$$
\begin{aligned}
\left(\mathbf{y}, \mathbf{y}\left(\langle z p\rangle+\langle z p\rangle^{*}\right)[I-J]\right) & =-\left(\mathbf{y} R, \mathbf{y}\left(\langle z p\rangle+\langle z p\rangle^{*}\right)[I-J]\right) \\
& =\overline{\left(\mathbf{y}, \mathbf{y}\left(\langle z p\rangle+\langle z p\rangle^{*}\right) R[I+J]\right)} \\
& =\overline{\left(\mathbf{y}, \mathbf{y}\left(\langle z p\rangle^{*}+\langle z p\rangle\right)[I+J]\right)} .
\end{aligned}
$$

Thus

$$
\begin{aligned}
\frac{d}{d t} \operatorname{Neh}\left(f_{t}(\mathbf{D})\right) & =\frac{1}{2} \operatorname{Re}\left[\left(\mathbf{y}, \mathbf{y}\left[\langle z p\rangle+\langle z p\rangle^{*}\right][I-J]\right)+\left(\mathbf{y}, \mathbf{y}\left(\langle z p\rangle^{*}+\langle z p\rangle\right)[I+J]\right)\right] \\
& =\operatorname{Re}\left(\mathbf{y}, \mathbf{y}\left[\langle z p\rangle+\langle z p\rangle^{*}\right]\right)=2 \operatorname{Re}(\mathbf{y}, \mathbf{y}\langle z p\rangle) .
\end{aligned}
$$

Corollary 4.11. Let $x \in \mathscr{D}_{-n}^{n}$ satisfy $x_{k}=-\overline{x_{-k}}, k=1, \ldots, n$. Under the hypotheses of the previous theorem,

$$
\left.\frac{d}{d t}\right|_{t=t_{0}} \operatorname{Neh}\left(\mathbf{y}_{t}\right)=\operatorname{Re} \lim _{r \rightarrow 1^{-}} \frac{1}{\pi i} \int_{\gamma_{r}}\left(z y_{t_{0}}^{\prime}(z)\right)^{2} p_{t_{0}}(z) \frac{d z}{z}
$$


Proof. Note that $\left(z y^{\prime}\right)^{*}=-z\left(y^{*}\right)^{\prime}$. By Corollaries 3.2 and 4.10, we have that

$$
\begin{aligned}
\frac{d}{d t} \operatorname{Neh}(\mathbf{y}) & =\lim _{r \rightarrow 1^{-}} 2 \operatorname{Re} \frac{1}{2 \pi i} \int_{\partial \mathbf{D}}\left(z y^{\prime}\right)^{*}(z) \cdot\left(z y^{\prime}\right)(z) \cdot p(z) \frac{d z}{z} \\
& =-\lim _{r \rightarrow 1^{-}} \operatorname{Re} \frac{1}{\pi i} \int_{\partial \mathbf{D}} z y^{* \prime}(z) \cdot z y^{\prime}(z) \cdot p(z) \frac{d z}{z} \\
& =\lim _{r \rightarrow 1^{-}} \operatorname{Re} \frac{1}{\pi i} \int_{\partial \mathbf{D}} z^{2} y^{\prime}(z)^{2} \cdot p(z) \frac{d z}{z} .
\end{aligned}
$$

Thus the derivative of the Nehari functional, at points where $f$ is admissible for the quadratic differential $x^{\prime 2} d z^{2}$, is naturally associated to the pull-back

$$
y^{\prime}(z)^{2} d z^{2}=\left(x^{\prime} \circ f(z)\right)^{2} f^{\prime}(z)^{2} d z^{2}
$$

of $x$ under $f$.

In particular, if we denote

$$
\frac{Q_{t}(z)}{z^{2}} d z^{2}=y^{\prime}(z)^{2} d z^{2}
$$

and

$$
Q_{t}(z)=\sum_{k=-2 n}^{2 n} d_{k} z^{k}
$$

then

$$
\left.\frac{d}{d t} \operatorname{Neh}\left(\mathbf{y}_{t}\right)\right|_{t=t_{0}}=2 \operatorname{Re} \sum_{k=0}^{2 n} d_{-k} p_{k} .
$$

Remark 4.12. Note that what we have computed above is the functional derivative, not just the derivative with respect to the time parameter. That is, Theorem 4.9, Corollary 4.10 and Corollary 4.11 hold whether or not $p_{t}$ is the generator of the extremal one-parameter flow for $t \geq t_{0}$.

\section{Concluding remarks}

First, we observe that Corollary 4.11 specifies a natural pairing of quadratic differentials and infinitesimal generators of the Friedland-Schiffer equation. One may think of the operator

$$
z p(z) \frac{\partial}{\partial z}
$$

as the infinitesimal generator. The result of contracting a quadratic differential with an infinitesimal generator is an abelian differential:

$$
\frac{Q(z)}{z^{2}} d z^{2}\left(z p(z) \frac{\partial}{\partial z}\right)=\frac{Q(z)}{z} p(z) d z .
$$

Integrating this over the circle results in a natural pairing: for $\gamma_{r}$ the positively oriented curve $|z|=r$ we have for any $Q$ and $p$ the number

$$
\lim _{r \rightarrow 1^{-}} \frac{1}{2 \pi i} \int_{\gamma_{r}} \frac{Q(z)}{z} p(z) d z
$$

which for $Q(z) / z^{2}=y^{\prime}(z)^{2}$ agrees with the right hand side of the expression in Corollary 4.11 up to a multiplicative constant. 
Remark 5.1. This association of quadratic differentials with a dual space of infinitesimal generators (in fact, the dual of the positive part of the Witt algebra [7, Remark 4]) appears naturally in the Loewner-Schiffer system for a quadratic differential arising from the combination of Schiffer variation with Loewner theory [4]. See [7, Theorem 2].

Next, we make some observations about the infinitesimal expression of the case of equality. Assume that some domain $D_{1}$ is extremal for the Nehari functional. We saw in Section 4.2 that $D_{1}$ is $\mathbf{D}$ minus some system of trajectories of the quadratic differential $x^{\prime}(z)^{2} d z^{2}$. Since the Nehari functional increases as the domain increases, and $\operatorname{Neh}\left(D_{1}\right)=0$, it follows that if $D_{2}$ is obtained from $D_{1}$ by truncating some or all of the arcs (i.e. $D_{2}$ is chosen such that $D_{1} \subset D_{2} \subset \mathbf{D}$ ) then $\operatorname{Neh}\left(D_{2}\right)=0$. Thus one obtains a family of extremals by arc truncation.

On the other hand, assume that $f_{t_{0}}$ is extremal for the functional. If $f_{t}$ is a one-parameter family of maps solving the Friedland-Schiffer equation with initial condition $f_{t_{0}}$ at $t=t_{0}$, and $f_{t}$ extremal for the Nehari functional on some interval $\left[t_{0}, a\right)$, then we know that $f_{t}$ must continue to be a slit map. We can thus assume that $p_{t}$ has the special form

$$
p_{t}(z)=\frac{\kappa(t)+z}{\kappa(t)-z}
$$

for some $\kappa$ of unit modulus. Denote

$$
Q_{t}(z)=z^{2} y_{t}^{\prime}(z)^{2}=\sum_{k=-2 n}^{2 n} d_{k} z^{k} .
$$

Since $y_{-k}=-\bar{y}_{k}$, we have that $d_{k}=\bar{d}_{-k}$ and $d_{0}$ is real. (Note that both $d_{k}$ and $y_{k}$ depend on t.) For $\gamma_{r}$ the circle $|z|=r$

$$
\begin{aligned}
\frac{d}{d t} \operatorname{Neh}\left(\mathbf{y}_{t}\right) & =\lim _{r \rightarrow 1^{-}} \operatorname{Re} \frac{1}{\pi i} \int_{\gamma_{r}} Q_{t}(z) p_{t}(z) \frac{d z}{z}=2 d_{0}+4 \operatorname{Re} \sum_{1}^{2 n} d_{-k} \overline{\kappa(t)}^{k} \\
& =2 d_{0}+2 \sum_{-2 n}^{-1} d_{k} \overline{\kappa(t)}^{k}+2 \sum_{-2 n}^{-1} \overline{d_{k}} \kappa(t)^{k}=2 \sum_{k=-2 n}^{2 n} d_{k} \overline{\kappa(t)}^{k}=2 Q_{t}(\overline{\kappa(t)}) .
\end{aligned}
$$

Thus we see that, at an $f_{t}$ which is admissible for $x^{\prime 2} d z^{2}$, the derivative of the Nehari functional is zero if and only if $\overline{\kappa(t)}$ is a zero of $Q_{t}(z)=z^{2} y_{t}^{\prime}(z)^{2} d z^{2}$.

This has the following geometric interpretation. Either $x^{\prime}\left(f_{t}(\bar{\kappa})\right)=0$, in which case $f_{t}(\bar{\kappa})$ is a zero of the quadratic differential $x^{\prime}(z)^{2} d z^{2}$, or $f_{t}^{\prime}(\bar{\kappa})=0$, in which case $\bar{\kappa}$ maps to the tip of an arc of the boundary of $D_{1}$. Thus the condition that $Q(\bar{\kappa})=0$ means that either $f_{t}$ continues to lengthen an existing trajectory of $x^{\prime}(z)^{2} d z^{2}$ or begins a new fork at a zero of $x^{\prime}(z)^{2} d z^{2}$.

Finally we observe that these results are an example of what is sometimes called Teichmüller's principle, which says that a functional is associated in general with a quadratic differential. Here, Teichmüller's principle manifests itself in two ways. First, given a quadratic differential, we obtain a specific functional by Nehari's method (Theorem 4.4). Second, the derivative of the functional at an extremal is given by a quadratic differential (Theorem 4.9 and Corollary 4.11), which is a general consequence of Schiffer's variational method. Thus, first a choice of quadratic differential determines a functional, and then the derivative of the functional 
in turn determines a quadratic differential. The point of interest is that Corollary 4.11 demonstrates that the resulting quadratic differential is the pull-back of the original quadratic differential under the extremal map.

\section{References}

[1] Friedland, S., and M. Schiffer: On coefficient regions of univalent functions. - J. Anal. Math. 31, 1977, 125-168.

[2] Jавотіnsky, E.: Representation of functions by matrices. Application to Faber polynomials. Proc. Amer. Math. Soc. 4, 1953, 546-553.

[3] Nehari, Z.: Some inequalities in the theory of functions. - Trans. Amer. Math. Soc. 75, 1953, $256-286$.

[4] Rотн, O.: Pontryagin's maximum principle in geometric function theory. - Complex Var. Theory Appl. 41:4, 2000, 391-426.

[5] Roтн, O.: Control theory in $H(\mathbf{D})$. - Ph.D. thesis, Würzburg, 1998; Tectum Verlag, Marburg, 1998.

[6] Schiffer, M., and O. Tammi: A Green's inequality for the power matrix. - Ann. Acad. Sci. Fenn. Ser. A I Math. 501, 1971.

[7] Schippers, E.: The power matrix, coadjoint action and quadratic differentials. - J. Anal. Math. 98, 2006, 249-277.

[8] Schippers, E.: A power matrix approach to the Witt algebra and Loewner equations. arXiv:CV/0907.1601.

Received 4 August 2009 